\title{
Atoms in superintense laser fields
}

\author{
C. A. S. Lima and L. C. M. Miranda \\ Departamento de Física, Universidade de Brasília, 70.910-Brasília, D. F. Brazil
}

(Received 28 July 1980)

\begin{abstract}
A new calculational ansatz for the quantum problem of a hydrogenlike atom in a nonresonant superintense laser field is presented. It is shown that the ground-state energy scales down from the field-free value by the ratio of the Bohr radius to the electron oscillation amplitude in the laser field.
\end{abstract}

The description of atomic bound states in the presence of an intense nonresonant laser field has attracted the attention of a number of authors..$^{1-8}$ In these works, the simple model where one assumes a hydrogenlike atom and treats the laser beam as a classical plane electromagnetic wave of frequency $\omega$, in the dipole approximation, is usually considered. The Schrödinger equation for the electronic wave function is then

$$
i \hbar \dot{\psi}(\overrightarrow{\mathbf{r}}, t)=\left[\frac{1}{2 m}\left(\hat{p}+\frac{e}{c} \overrightarrow{\mathrm{A}}(t)\right)^{2}+V(\overrightarrow{\mathbf{r}})\right] \psi(\overrightarrow{\mathbf{r}}, t),
$$

where $\overrightarrow{\mathrm{A}}(t)=A(\hat{x} \cos \omega t+\hat{y} \sin \omega t)$ is the vector potential for a circularly polarized laser beam and $V(\overrightarrow{\mathbf{r}})=-Z e^{2} /|\overrightarrow{\mathbf{r}}|$ is the atomic binding potential. Unfortunately, no exact solutions to Eq. (1) are currently available. Nevertheless, there exists a wide range of laser intensities (up to a critical intensity $I_{c}-10^{11} \mathrm{~W} / \mathrm{cm}^{2}$ for a $\mathrm{Nd}$ : glass laser) for which approximate, nonperturbative methods are applicable. Among these methods the most widely used are the Keldysh approximation, the spatial translation, ${ }^{3,6}$ and the momentum translation. ${ }^{4}$ We refer to Ref. 6 for a detailed comparison between the relative validity and applicability of these various methods. For laser intensities much larger than $I_{c}$ the above methods are no longer valid.

In this paper we present a new approach describing the bound states of Eq. (1) applicable to laser intensities $I$ such that the amplitude of the electron oscillation in the laser field $a=e A / \mathrm{cm} \omega$ is much greater than the size of the bound system $a_{0}=\hbar^{2}$ / $Z e^{2} m$ namely, $I \gg I_{c}$ where $I_{c}=\mathrm{cm}^{2} a_{0}^{2} \omega^{4} / 4 \pi e^{2}$. We call this intensity range the superintense laser regime. It is shown that a variational solution to Eq. (1), though still hydrogenlike in character, now exhibits a parametric dependence in the laser-field parameter $(a)$. This leads to a modified electron radial distribution function which now peaks at a position $(r \sim a)$ that shifts monotonically to higher values with the laser intensity. To show how this comes about we begin by performing a canonical transformation in Eq. (1),,$^{3,6}$ i.e.,

$$
\psi(r, t)=e^{i \vec{b}(t) \cdot \hat{p} / \hbar} e^{i \eta(t) / \hbar} \phi(\overrightarrow{\mathrm{r}}, t),
$$

where

$$
\vec{\delta}(t)=-\frac{e}{m c} \int^{t} d t^{\prime} \overrightarrow{\mathrm{A}}\left(t^{\prime}\right), \quad \eta(t)=-\frac{e^{2}}{2 m c^{2}} \int^{t} d t^{\prime} A^{2}\left(t^{\prime}\right) .
$$

In this manner, we transfer the laser-field dependence from the kinetic-energy term to the potential-energy term, namely,

$$
i \hbar \dot{\phi}(\overrightarrow{\mathbf{r}}, t)=\left(\frac{\hat{p}^{2}}{2 m}+V(\overrightarrow{\mathbf{r}}-\vec{\delta}(t))\right) \phi(\overrightarrow{\mathbf{r}}, t) .
$$

In this new representation the electronic wave function is given by the solution to the Schrödinger equation for an atom in which the electron oscillates with the laser frequency and amplitude $\vec{\delta}(t)$. The usual spatial-translation approximation ${ }^{3,6}$ consists in completely neglecting the shift $\vec{\delta}(t)$ in the potential-energy term of Eq. (4) and, therefore, $\phi(\vec{r}, t)$ is taken as the solution of the fieldfree problem. As discussed in Ref. 6 this approximation is justifiable provided $|\vec{\delta}(t)|=a$ is much smaller than the size of the bound system. An improvement over this approximation consists of expanding $V(\overrightarrow{\mathbf{r}}-\vec{\delta})$ as $V(\overrightarrow{\mathbf{r}})-(\vec{\delta} \cdot \vec{\nabla}) V(\overrightarrow{\mathbf{r}})+\cdots$, and treat the second term of this expansion as a perturbation to the field-free problem. This procedure leads essentially to the same results as those of the Keldysh approximation. ${ }^{1,6}$

Let us now consider the problem set in Eq. (4). The laser-dressed binding potential $-Z e^{2} /|\overrightarrow{\mathbf{r}}-\vec{\delta}|$ can be written as

$$
\begin{aligned}
V & =-\frac{Z e^{2}}{\left(r^{2}+a^{2}\right)^{1 / 2}}\left(1-\frac{2 \overrightarrow{\mathrm{r}} \cdot \vec{\delta}}{\bar{r}^{2}+a^{2}}\right)^{-1 / 2} \\
& \simeq-\frac{Z e^{2}}{\left(r^{2}+a^{2}\right)^{1 / 2}}\left(1-\frac{\overrightarrow{\mathrm{r}} \cdot \vec{\delta}}{r^{2}+a^{2}}+\cdots\right) .
\end{aligned}
$$

We should note the rapidly converging character of expansion (5) since $\left(\overrightarrow{\mathrm{r}} \cdot \vec{\delta} / r^{2}+a^{2}\right)^{n}$ will never exceed $\left(\frac{1}{2}\right)^{n}$. Thus we can safely assume that the binding potential is adequately described by the first term, namely, $V=-Z e^{2}\left(r^{2}+a^{2}\right)^{-1 / 2}$. So far, the approximation made has no bearing as to the 
laser-field strengths. Hence, in the above form the binding potential is suitable for the description of an atom under either an intense or a weak laser field. However, should one wish to go a step further by including the terms in $\vec{r} \cdot \vec{\delta}$, this can easily be accomplished in a way similar to Ref. 6 previously mentioned. The resulting wave function will differ from the zeroth-order result by essentially a Keldysh-type sinusoidal phase factor.

The solution to Eq. (4) for $V \simeq Z e^{2} /\left(r^{2}+a^{2}\right)^{1 / 2}$ will now be calculated by resorting to the variational method. To be precise, we will look in the following for the ground-state solution to Eq. (4). To this end we choose as a trial function a hydrogenlike ground-state wave function, namely,

$$
\Phi=A e^{-r \beta},
$$

where $A$ is a normalization constant and $\beta$ is the variational parameter obtained from the condition that

$$
\left\langle\Phi\left|\frac{\hat{p}^{2}}{2 m}-\frac{Z e^{2}}{\left(r^{2}+a^{2}\right)^{1 / 2}}\right| \Phi\right\rangle
$$

is a minimum with respect to $\beta$. The results from computer-aided calculations for the groundstate energy $\epsilon_{0}$ and the variational parameter $\beta$, for several laser-power settings are summarized in Table I. In our calculations we have introduced the parameters $\lambda=a / a_{0}$ as a measure of the laserfield strength, and $\epsilon_{0}$ as a measure of the groundstate energy $E_{0}$ in units of $Z e^{2} / a$, i.e., $E_{0}=-Z e^{2} \epsilon_{0} /$ $a$. It follows from $T$ able $I$ that as the laser-field strength increases, both $\epsilon_{0}$ and $\beta a$ increase, reaching essentially constant values beyond certain laser-field strength $(\sim \lambda=50)$. In other words, at sufficiently large laser intensities (i.e., $a \gg a_{0}$ ), $\beta \sim a^{-1}$, and $E_{0} \sim a^{-1}$. This entails that the effect of a superintense laser field is to weaken the electron binding to the nucleus. This situation is depicted in Fig. 1 where we plot both the laserdressed Coulomb potential as well as the radial electronic distribution function obtained from Eq. (6) for different values of the laser-field strength. The above result regarding the effect of a nonresonant superintense laser field remains unalter-

TABLE I. Dependence of the ground-state length parameter $(\beta)$ and energy $\left(\epsilon_{0}\right)$ on the laser-field strength $(\lambda)$. The parameter $\lambda$ is related to the laser intensity

\begin{tabular}{|c|c|c|c|c|}
\hline & 5 & 10 & 50 & $\geqslant 100$ \\
\hline$\beta a$ & 0.82 & 0.98 & 1.16 & 1.20 \\
\hline$\epsilon_{0}$ & 0.15 & 0.18 & 0.24 & 0.27 \\
\hline
\end{tabular}
in $\mathrm{W} / \mathrm{cm}^{2}$ by $\lambda=6.5 \times 10^{24} \omega^{-2} I^{1 / 2}\left(\mathrm{~W} / \mathrm{cm}^{2}\right)$.

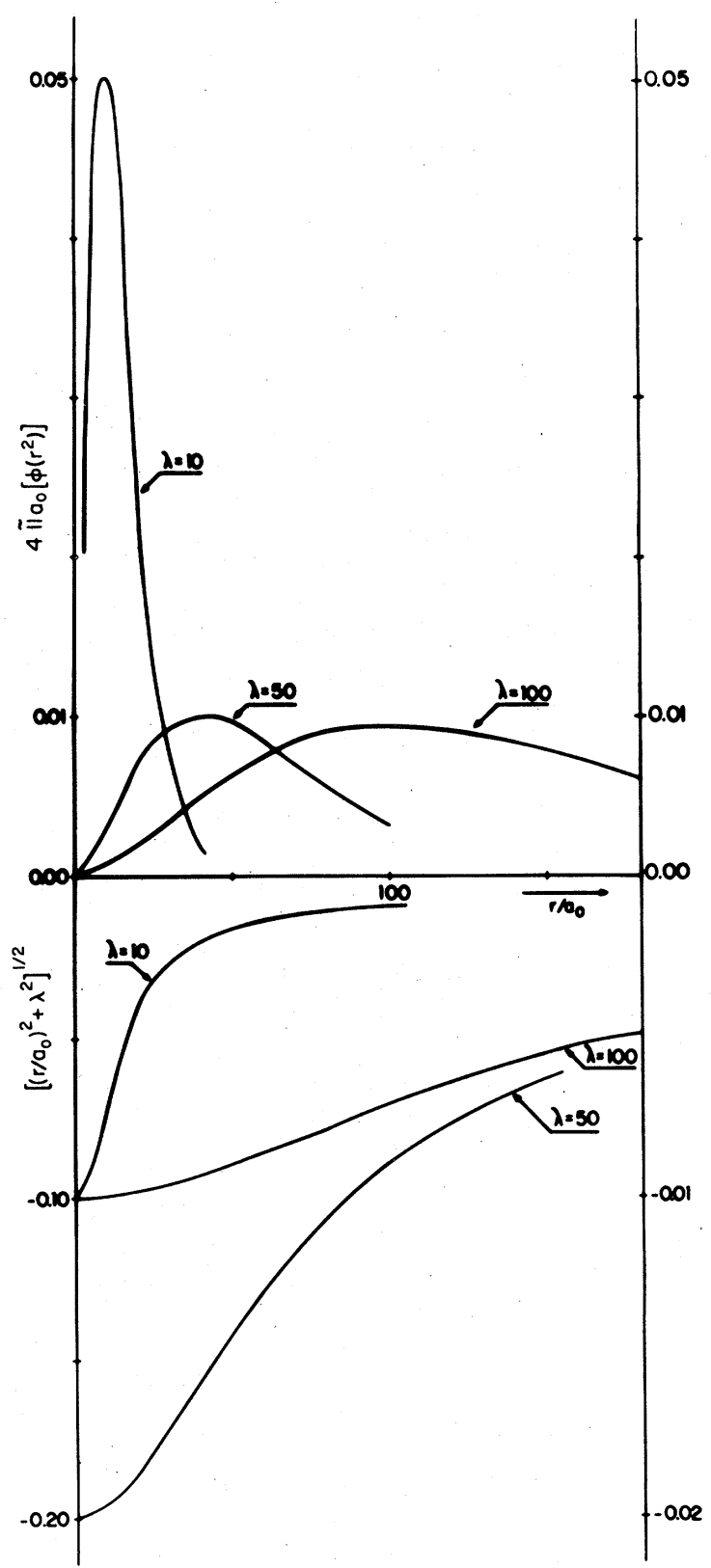

FIG. 1. The radial distribution function and the laserdressed potential energy for different values of the laser-field-strength parameter $\lambda$. The energy values corresponding to the curves labeled $\lambda=50$ and $\lambda=100$ are given in the right-hand vertical scale.

ed if one uses different trial functions. This was demonstrated by also using a $1 s$ harmonic-oscillator wave function as a second trial function. However, with this second choice for the trial functions the ground-state energy we got was consistently larger than that for the hydrogenic wave function which shows that the latter is a best choice. We should further notice that objections can be raised 
to our using a single-basis wave function in Eq. (6). The problem of deciding how complex a trial function should be has been addressed in Refs. $9-12$. For instance, in Ref. 9 the inclusion of a $2 s$ hydrogenic wave function produced a $20 \%$ better estimate for the ground-state energy for a hydrogen atom in an intense dc magnetic field. A more realistic approach should use a more complex basis as, for instance, the Reinhardt ${ }^{10-12}$ expansion in the basis of $L^{2}$ Laguerre-type functions. Improvements of this kind are definitely important for quantitative discussions but do not alter the qualitative predictions made in regard to the physical behavior of the atomic system based on single-basis trial functions. Since our main purpose in this paper is to bring about a new way for investigating the bound states for an atom in the presence of a superintense laser field [e.g., via the laser dressed potential of Eq. (5)] we feel that Eq. (6) is adequate enough.

In conclusion some comments regarding the range of nonresonant laser intensities within which our model is valid should be made. A lower limit has already been established as the intensity $I_{c}$
$=2.6 \times 10^{-50} \omega^{4} Z^{-2}\left(\mathrm{~W} / \mathrm{cm}^{2}\right)$ which resulted from requiring $a_{L}$ to be much larger than the size of our bound system in the field-free limit $\left(a \gg a_{0}\right)$. Now as to the existence of an upper limit $I_{u}$, the point to be considered is that our model holds as long as the conditions for using the dipole approximation to describe the laser field obtain. This means that, whatever the laser intensity $I$, the length parameter for our laser-dressed atom should always remain much smaller than the laser wavelength. This implies that an upper limit does exist, $I_{u}$ $=3.7 \times 10^{-11} \omega^{2}\left(\mathrm{~W} / \mathrm{cm}^{2}\right)$. As it turns out, however, this limit is more of an academic interest than an actual restriction, the point being that $I_{u}$ still lies comfortably above the highest intensities available today and in a foreşeeable future, for those lasers of practical interest. For example, in the case of a $\mathrm{CO}_{2}$ laser $\left(\omega=1.9 \times 10^{14} \mathrm{~s}^{-1}\right)$ and for $Z=1$ our model is applicable for laser intensities lying in the range $3.3 \times 10^{7} \ll I \ll 1.3 \times 10^{18}\left(\mathrm{~W} / \mathrm{cm}^{2}\right)$.

We gratefully acknowledge profitable discussions with Dr. A. Kiel from UNICAMP.
${ }^{1}$ L. V. Keldysh, Zh. Eksp. Teor. Fiz. 46, 1090 (1964) [Sov. Phys. - JE TP 20, 1307 (1965)].

${ }^{2}$ H. B. Bebb and A. Grold, Phys. Rev. 143, 1 (1966).

${ }^{3}$ W. C. Henneberger, Phys. Rev. Lett. 21,838 (1968).

${ }^{4}$ H. R. Reiss, Phys. Rev. D $\underline{4}, 3533$ (1971).

${ }^{5}$ A. M. F. Lau, Phys. Rev. A $18,172(1978)$ and references therein.

${ }^{6}$ H. S. Brandi, B. Koiller, H. G. P. Lins de Barros, L. C. M. Miranda, and J. J. Castro, Phys. Rev. A 17, 1900 (1978).
${ }^{7}$ M. Jain and N. Tzoar, Phys. Rev. A 15, 147 (1977).

${ }^{8}$ M. H. Mittleman, Phys. Lett. $47 \mathrm{~A}, 55$ (1974).

${ }^{9}$ A. K. Rajagopal, G. Chanmugan, R. F. O'Connell, and G. L. Sumerlian, Astrophys. J. 177, 713 (1972).

${ }^{10}$ E. J. Heller, W. P. Reinhardt, and H. A. Yamani, J. Comput. Phys. 13, 536 (1973).

${ }^{11}$ E. J. Heller, T. N. Reseigno, and W. P. Reinhardt, Phys. Rev. A 8, 2946 (1973).

${ }^{12}$ H. A. Yamani and W. P. Reinhardt, Phys. Rev. A 11 , 1145 (1975). 